\title{
Chewing as a forming application: A viscoplastic damage law in modelling food oral breakdown
}

Cite as: AIP Conference Proceedings 1896, 150002 (2017); https://doi.org/10.1063/1.5008170

Published Online: 16 October 2017

C. G. Skamniotis, M. N. Charalambides, and M. Elliott

\section{ARTICLES YOU MAY BE INTERESTED IN}

On modeling the large strain fracture behaviour of soft viscous foods

Physics of Fluids 29, 121610 (2017); https://doi.org/10.1063/1.4993754

Mechanical characterization and micromechanical modeling of bread dough

Journal of Rheology 57, 249 (2013); https://doi.org/10.1122/1.4768463

Extrusion of unleavened bread dough: Experiments and simulations

Journal of Rheology 61, 49 (2017); https://doi.org/10.1122/1.4966692

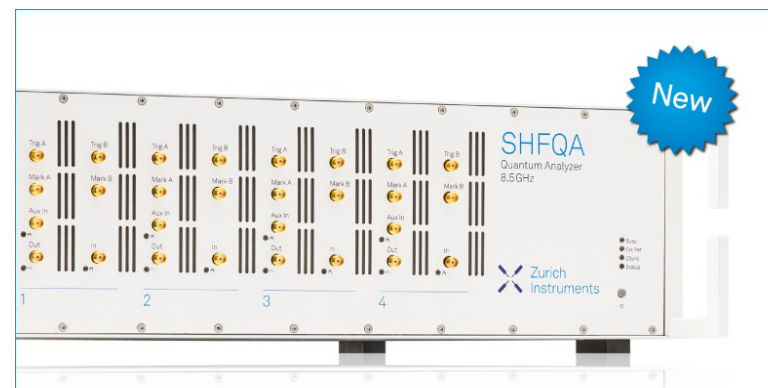

Your Qubits. Measured.

Meet the next generation of quantum analyzers

- Readout for up to 64 qubits

- Operation at up to $8.5 \mathrm{GHz}$, Find out more

- mixer-calibration-free

- Signal optimization with minimal latency 


\title{
Chewing as a forming application: A viscoplastic damage law in modelling food oral breakdown
}

\author{
C.G. Skamniotis 1 , M.N. Charalambides ${ }^{1}$, M. Elliott ${ }^{\text {a }}$ \\ ${ }^{1}$ Department of Mechanical Engineering, Imperial College London \\ London SW7 2AZ, United Kingdom \\ E-mail: c.skamniotis13@imperial.ac.uk,m.charalambides@imperial.ac.uk \\ ${ }^{a}$ Mars Petcare UK Ltd, Leicestershire, UK
}

\begin{abstract}
The first bite mechanical response of a food item resembles compressive forming processes, where a tool is pressed into a workpiece. The present study addresses ongoing interests in the deformations and damage of food products, particularly during the first bite, in relation to their mechanical properties. Uniaxial tension, compression and shear tests on a starch based food reveal stress-strain response and fracture strains strongly dependent on strain rate and stress triaxiality, while damage mechanisms are identified in the form of stress softening. A pressure dependent viscoplastic constitutive law reproduces the behavior with the aid of ABAQUS subroutines, while a ductile damage initiation and evolution framework based on fracture toughness data enables accurate predictions of the product breakdown. The material model is implemented in a Finite Element (FE) chewing model based on digital pet teeth geometry where the first bite of molar teeth against a food item is simulated. The FE force displacement results match the experimental data obtained by a physical replicate of the bite model, lending weight to the approach as a powerful tool in understanding of food breakdown and product development.
\end{abstract}

\section{INTRODUCTION}

Amongst common material forming applications, chewing can be considered to be one, since it involves multi-axial stresses due to teeth indentation, various strain rates and non-linear geometries (1). Towards, product design and optimization, predictions of how the food will perform during oral processing are desirable by the food industry (2). However, sensory attributes may not be well predicted by only measuring food mechanical properties as mastication involves varying parameters across consumers, such as different speeds, teeth shape, bite forces, jaw motions, as well as friction (3). In contrast, a Finite Element mastication model raises greater potential for reconstructing the real phenomenon (1) and this is considered in the present study. Such a study requires advanced material modelling techniques, including suitable constitutive laws for food products, which are often highly compliant non-linear and strongly time dependent (4, 5), combined with progressive damage schemes to predict fracture (1). All these capabilities induce non-linearity in the FE computations which complicate the numerical analysis (6). Further implications are induced by complex contacts involved in chewing, particularly for tough foods, which requires significant computation time and often causes convergence problems. This study however shows that modelling such complex phenomena is possible by using explicit FE solvers (6) and proper mesh design. Although much work has been reported on characterising and modelling the response of time dependent materials, and particularly foods $(4,5,7)$, only recently the authors utilized such models in an FE reconstruction of mastication (1). A few studies report on the stress fields generated in the teeth (8-10) as well as in the food item (11-13) during oral processing, yet none accounted for the usual rate dependent nature of foods, neither for the fracture toughness parameter nor the interfacial frictional conditions. It has been shown that smoothed particle hydrodynamics (SPH) is an attractive method for modelling large deformations (11-13), as most of the foods experience this during chewing, albeit the computational time had to be sacrificed. In contrast, this study presents a realistic model of chewing in pets, accounting for the true mechanical behaviour of tough foods including fracture toughness, the complex geometries of the teeth as well as friction. Such FE analyses assist in understanding of the food breakdown mechanisms and reflect industry needs for time and cost efficient techniques towards product development and optimisation (1).

\section{EXPERIMENTAL AND MODELLING}

The starch based food includes short fibres and proteins forming a fibre reinforced bio-polymer with an amorphous matrix (1). Rectangular and dumbbell specimens were shaped from the starch extrudates, to facilitate uniaxial compression and tensile tests, respectively, using a universal testing machine. Additional rectangular samples were prepared for pure shear tests, using the shear sandwich configuration attached on a Dynamic Mechanical Analysis (DMA) machine. These data were obtained to compare the equivalent stress strain response between compression, shear and tension. Starch displays an incompressible behavior; the Poisson's ratio, $v$, was measured as 0.5 in a previous study (1). Figures $1 \mathrm{a}$ and $1 \mathrm{~b}$ show the compressive and tensile stress strain response until ultimate failure. Although in compression no sample separation occurred, after a certain strain level axial cracks were observed in the perimeter of the samples, attributed to the associated tensile strains $(v=0.5)$. These strains correspond to the maximum stress strain data points plotted in Figure 1a. Thereafter a stress drop was computed and thus the stress-strain was not considered valid beyond this point. The pure shear data were obtained for the maximum strain of 0.1 ; the corresponding equivalent stress-strain behavior for three strain rates $(0.5 / \mathrm{s}, 0.05 / \mathrm{s}, 0.005 / \mathrm{s})$ lied between the tensile and compressive data (data not shown here).

Proceedings of the 20th International ESAFORM Conference on Material Forming AIP Conf. Proc. 1896, 150002-1-150002-4; https://doi.org/10.1063/1.5008170 Published by AIP Publishing. 978-0-7354-1580-5/\$30.00 
A non-linear rate dependent behavior is observed as well as time dependent strain at break. No distinct yield point can be identified, while the material showed significant viscoelastic recovery after the tests. However, the viscoplastic model exactly reproduces the monotonic loading behavior by implementing a small initial elastic regime followed by a ratedependent yield behavior (Figure 1a, 1b) (1). Notably, the stresses are higher in tension than in compression, for the same corresponding strains, displaying the typical behavior of filled rubbers (6). Since the viscoplastic definition was adopted, such a phenomenon was treated as a pressure dependent yield behavior (14). The latter is modelled via implementing the ABAQUS Explicit user defined field subroutine (VUSDFLD) (6), which enables the user to calculate the stress triaxiality, $\eta$, for every integration point in the simulation, according to

$$
\eta=-p / q
$$

where, $p$, the pressure stress and, $q$, the Von Mises stress (1). The model is therefore constructed by providing tabular data of equivalent plastic strain and stress for each of the plastic strain rates tested and for each stress triaxiality; in this case, $\eta=-1 / 3$ and $\eta=1 / 3$, for uniaxial compression and uniaxial tension, respectively. Note that the model uses linear interpolation to predict the behavior for intermediate stress states, such as pure shear $(\eta=0)$, which is consistent with the shear test data here. The plastic strain at step, $i$, is computed based on the total and elastic strains as:

$$
\varepsilon_{\text {plastic }}^{i}=\varepsilon_{\text {total }}^{i}-\varepsilon_{\text {elastic }}^{i}=\varepsilon_{\text {total }}^{i}-\frac{\sigma^{i}}{E}
$$

where, $E$, the elastic modulus, $\sigma$, the stress (6). The value of, $E=100 \mathrm{MPa}$, is deliberately given to minimize the elastic strains such that the plastic strain rate can be assumed equal to the total strain rate (1). The Poisson's ratio, $v$, was defined as 0.49 to avoid numerical problems associated with the Dynamic Explicit ABAQUS solver (6).
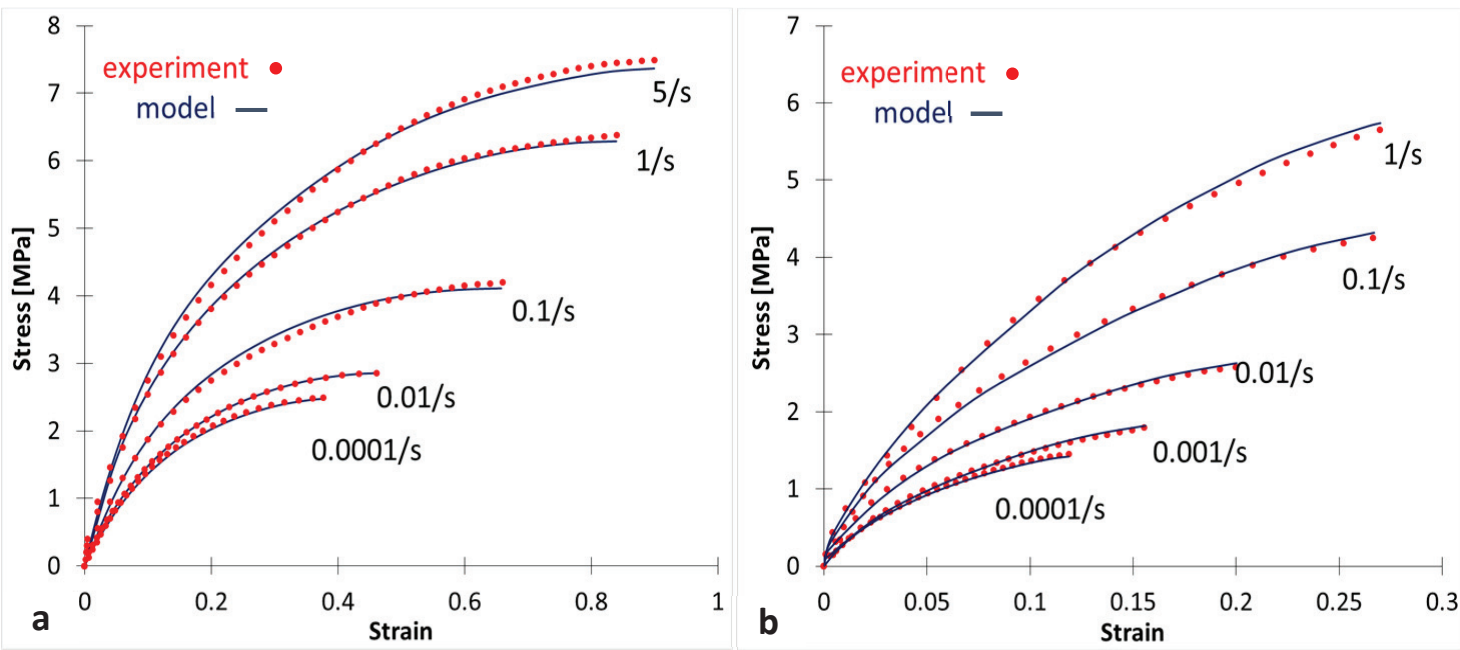

Figure 1. Uniaxial true stress - true strain experimental data and model fit, (a) compressive stress-strain data for the strain rates: $0.0001,0.01,0.1,1,5 / \mathrm{s}$, (b) tensile stress-strain data for the strain rates: 0.0001, 0.001, 0.01, 0.1, 1/s.

Fracture toughness data were obtained for starch in a previous study by the authors, using the Essential Work of Fracture and orthogonal cutting methods (1). Both methods yielded toughness values independent of crack speed. Our very recent work however examined a novel essential work of fracture scheme for highly dissipative materials, such as starch, on which a toughness of $0.94 \mathrm{~kJ} / \mathrm{m}^{2}$ was measured. This value assists in modelling the oral food breakdown via employing the element stiffness degradation scheme, which is a mesh independent measure commonly used to describe ductile damage in metals (1). The framework considers an equivalent strain based damage onset criterion depending on strain rate and stress triaxiality, followed by a fracture toughness based element stiffness degradation law until final element deletion. This model is advantageous over the cohesive traction separation laws, as it does not require a predefined crack path and the insertion of cohesive elements (15). Such capabilities are desired in chewing applications since complex crack patterns are anticipated, with no knowledge of their location.

In the numerical model, three upper (maxilla) and lower carnassial teeth (mandible) are considered, generated from Computed Tomography (CT) scans of a Boxer scull (Figures 2a, 2b) (1). The maxilla is fixed while the mandible is constrained in all translations and rotations apart from the $\mathrm{Y}$ axis, along which a maximum displacement of $14 \mathrm{~mm}$ is applied to reach the centric occlusion state (Figure 2c), with the speed of $16.6 \mathrm{~mm} / \mathrm{s}$. Penalty contact is enforced with a friction coefficient, $\mu=0.2$, obtained from sliding tests using similar interfaces, under dry conditions. The jaws are modelled as discrete rigid parts $(1,12)$. The ABAQUS Dynamic Explicit (6) is used, with applied mass scaling in order to reduce computation time. However, validity criteria were considered to ensure that the artificially added inertia does not influence the FE results $(1,6,7)$. Element distortion is a common concern in numerical problems involving high strains $(6,12)$ and therefore in this study care was necessary to avoid such effects. The latter was addressed by an 
appropriate mesh design, through elements of initially high aspect ratios, such that the compressive strains act along the long element dimension (6). The smallest element dimension was $0.06 \mathrm{~mm}$ while the food item was meshed with 852320 3D stress-displacement reduced integration hexahedral (C3D8R) elements. The jaws were meshed with 155079 rigid quadrilateral elements (R3D4). The simulation was run using an eight processor core (Intel i7 CPU $3.2 \mathrm{GHz}$ ). The numerical test is physically replicated by using 3D printed fixtures of the teeth as shown in Figure 2c. Stainless steel was used to give rise to the assumption of rigid teeth geometry in the FE model (1) (Figure 2c). A length of $20 \mathrm{~mm}$ was used for the cubic food product both in the FE model and in the experiment.
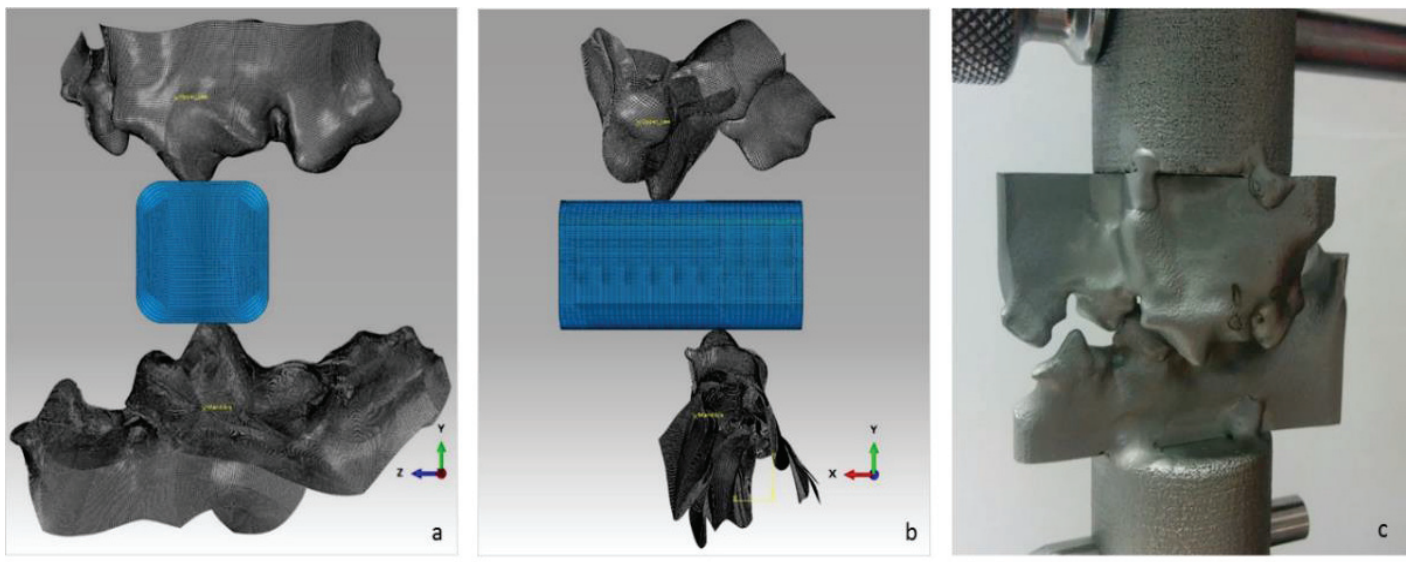

Figure 2. Mastication model of a boxer bite, a) FE model assembly (front view), b) FE model (side view), c) 3D printed jaw fixtures at centric occlusion state.

\section{RESULTS AND DISCUSSION}

The ability of the model in predicting food breakdown is demonstrated in Figure 3, where the experimental and simulation results fall close to each other both in terms of deformation contours and crack paths (Figures $3 \mathrm{a}, 3 \mathrm{~b}$ ), as well as in the force displacement response (shown in Figure 3c). The maximum force corresponds to crack onset in the form of element deletion, owing to tensile strains (1). Thereafter, fracture toughness determined the sample resistance against separation, underlining the importance of the toughness property in chewing models. As shown in Figure 3a total food separation did not occur, attributed to the tough nature of the starch based composite under investigation. This crack propagation regime corresponds to a mandible force drop, followed by an increase caused by compression of one of the two food fragments between the mandible and the palate surface (not shown in Figure 3a). The effect was not investigated as it depends on the food sample length used, while it may not reflect real chewing functions since food repositioning by the tongue naturally prevents such phenomena (12), towards chewing efficiency. Although sensitivity analysis displayed little effect of the friction coefficient on the forces (12) (results not shown), the effect was significant on the tensile strain and stress triaxiality field around the tooth indentation regions. A strongly non-uniform stress state field is observed due to the non-linear geometry of oral processing, involving multiaxial compression underneath the indentation tips, significant amount of shear in the core of the item, as well as biaxial tension mainly at its exterior faces. Non-uniform strain rates are also apparent; the range fells within the tested strain rates at which the material model was calibrated (1). The analysis serves in understanding deeply food breakdown, as well as in establishing relations between mechanical properties and sensory attributes $(1,12,13)$. The analysis lasted 5 hours, proving the FE method advantageous over the SPH. This allows for parametric studies to be performed towards product optimization, instead of physically producing trial products.
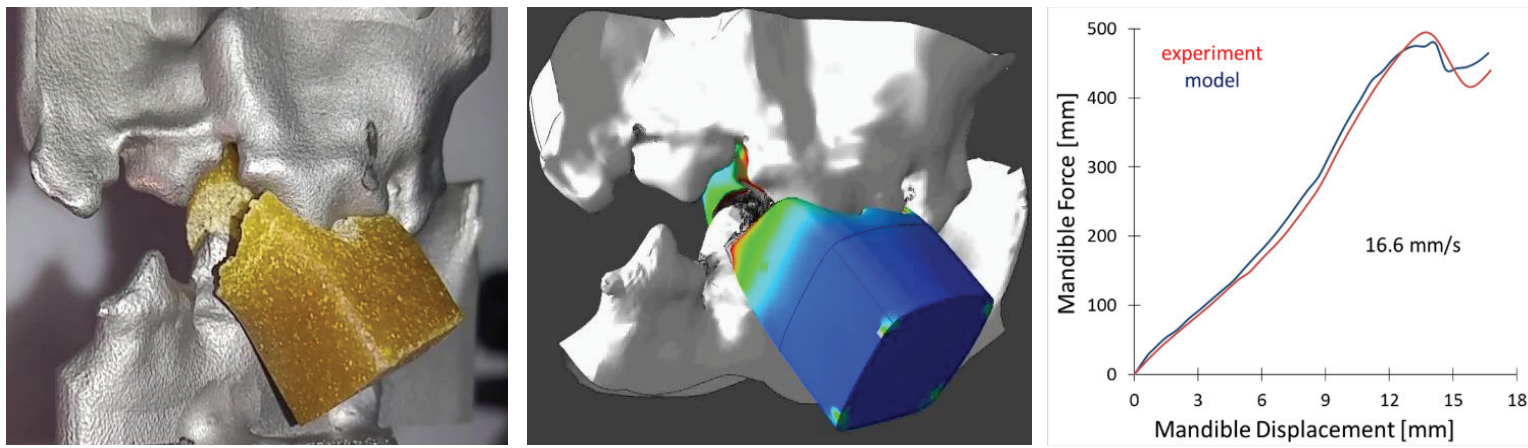

Figure 3. a) Chewing experiment, frame shows maximum displacement (centric occlusion), b) Chewing model at maximum displacement, c) Comparison between experimental and numerical mandible force versus displacement response. 


\section{ACKNOWLEDGEMENTS}

The authors would like to acknowledge Mars Petcare for the financial support and for providing the samples, and Dr. Michele Pettina for helping with the ABAQUS subroutine. In addition, Professor Gordon Williams is acknowledged for useful discussions.

\section{REFERENCES}

(1) Skamniotis C, Patel Y, Charalambides M, Elliott M. Fracture investigation in starch-based foods. Interface focus. 2016;6(3):20160005.

(2) Goh SM. An engineering approach to food texture studies: Imperial College London; 2002.

(3) Bierer TL. The role of biometrics in canine oral health and nutrition. Vet Med. 2007;1.

(4) Goh S, Charalambides M, Williams J. On the mechanics of wire cutting of cheese. Engineering fracture mechanics. 2005;72(6):931-46.

(5) Mohammed M, Tarleton E, Charalambides MN, Williams J. Mechanical characterization and micromechanical modeling of bread dough. Journal of Rheology (1978-present). 2013;57(1):249-72.

(6) Simulia D. ABAQUS 6.13 user's manual. Dassault Systems, Providence, RI. 2013.

(7) Mohammed M, Wanigasooriya L, Chakrabarti-Bell S, Charalambides M. Extrusion of unleavened bread dough: Experiments and simulations. Journal of Rheology (1978-present). 2017;61(1):49-65.

(8) Dejak B, Młotkowski A, Romanowicz M. Finite element analysis of stresses in molars during clenching and mastication. The Journal of prosthetic dentistry. 2003;90(6):591-7.

(9) Berthaume MA, Dumont ER, Godfrey LR, Grosse IR. The effects of relative food item size on optimal tooth cusp sharpness during brittle food item processing. Journal of The Royal Society Interface. 2014;11(101):20140965.

(10) Berthaume MA, Dumont ER, Godfrey LR, Grosse IR. How does tooth cusp radius of curvature affect brittle food item processing? Journal of The Royal Society Interface. 2013;10(84):20130240.

(11) Harrison SM, Eyres G, Cleary PW, Sinnott MD, Delahunty C, Lundin L. Computational Modeling of Food Oral Breakdown Using Smoothed Particle Hydrodynamics. Journal of Texture Studies. 2014;45(2):97-109.

(12) Harrison SM, Cleary PW. Towards modelling of fluid flow and food breakage by the teeth in the oral cavity using smoothed particle hydrodynamics (SPH). European Food Research and Technology. 2014;238(2):185-215.

(13) Harrison SM, Cleary PW, Eyres G, Sinnott MD, Lundin L. Challenges in computational modelling of food breakdown and flavour release. Food \& function. 2014;5(11):2792-805.

(14) Ward IM. Review: The yield behaviour of polymers. Journal of materials Science. 1971;6(11):1397-417.

(15) Mohammed I, Charalambides M, Williams J, Rasburn J. Modelling the microstructural evolution and fracture of a brittle confectionery wafer in compression. Innovative Food Science \& Emerging Technologies. 2014;24:48-60. 